\title{
ANALYSIS OF PARAMETRIC FLUORESCENCE AMPLIFIED IN A NONCOLLINEAR OPTICAL PARAMETRIC AMPLIFIER PUMPED BY THE SECOND HARMONIC OF A FEMTOSECOND Yb:KGW LASER
}

\author{
T. Stanislauskas, I. Balčiūnas, V. Tamuliene* ${ }^{\star}$ R. Budriūnas, and A. Varanavičius \\ Department of Quantum Electronics, Vilnius University, Sauletekio 10, LT-10223 Vilnius, Lithuania \\ E-mail: tomas.stanislauskas@ff.vu.lt
}

Received 29 September 2015, revised 18 November 2015; accepted 25 March 2016

\begin{abstract}
We present the results of theoretical and experimental investigation of amplified parametric fluorescence (APF) produced in a high-gain BBO-based femtosecond noncollinear optical parametric amplifier (OPA) pumped at $515 \mathrm{~nm}$. Differences of APF levels in Poynting vector walk-off-compensating and tangential phase-matching amplification geometries are examined. APF suppression due to the presence of a seed pulse in the OPA is measured and is found to be around 6 times in typical OPA operating conditions when pump-to-signal conversion efficiency is $11.5 \%$.
\end{abstract}

Keywords: parametric fluorescence, optical parametric amplifier, contrast

PACS: 42.65.Yj, 42.65.Re

\section{Introduction}

Optical parametric chirped-pulse amplification (OPCPA) [1] is a well-established method to produce high-energy, few-cycle pulses. This technique allows one to reach a gain of more than $10^{6}$ in a single pass of a few millimetre long crystal. However, this gain is attainable only by using high pump intensities in the amplifier crystal, which in turn increases the probability of efficient parasitic generation and amplification of optical parametric fluorescence. Consequently, amplified parametric fluorescence (APF) degrades signal stability and reduces extractable signal energy due to transfer of pump energy to the incoherent pedestal [2]. This lowers the compressed pulse contrast (ratio of the intensities of the amplified pulse and incoherent background), which is a major concern in high-field experiments. APF is only produced within the temporal window defined by the pump pulse, therefore it is proposed that using a short pump pulse in the first amplification stage, in which typically high gain is required, can increase pulse contrast at the OPCPA output [3]. The short-pulse preamplification approach was demonstrated in an OPCPA system driven by femtosecond ytterbium and picosecond neodymium pump sources [4].

\footnotetext{
* formerly Viktorija Pyragaite
}

APF is a result of parametric amplification of quantum noise due to two-photon emission from a virtual level excited by the intense pump field and stimulated by the signal and idler field zero-point fluctuations [5. 6. For a long time APF was successfully used as a seed source for tunable optical parametric amplifiers (OPAs) pumped by picosecond pulses 淐, 81. However, the output of OPAs of this type exhibits high shot-toshot pulse energy fluctuations, typically far exceeding those of the pump laser, possibly with individual shots entirely missing from the pulse train. From these high fluctuations one might infer that in the case of tight pump focusing, APF originates from a relatively low number of quantum noise photons, which, however, can be amplified to significant energies due to the high gain in parametric amplification systems. So far, works discussing the origin and amount of APF in OPA and OPCPA systems have not been numerous [9-14]. Efficient broad-band parametric amplification in the $\mathrm{BBO}$ crystal can be achieved by directing the signal beam at the so-called magic angle to the pump beam, which is about $2.5^{\circ}$ (inside the crystal) in the case of $515 \mathrm{~nm}$ pump. However, due to crystal birefringence the direction of the pump Poynting vector differs from that of the pump wave vector and the degree of spatial overlap between pump, signal, and idler beams depends on the orientation of the nonlinear crystal. In one orientation, the Poynting vector walk-off leads to better spatial overlap of pump and signal beams (see Fig. 1 (a)); this 
(a)
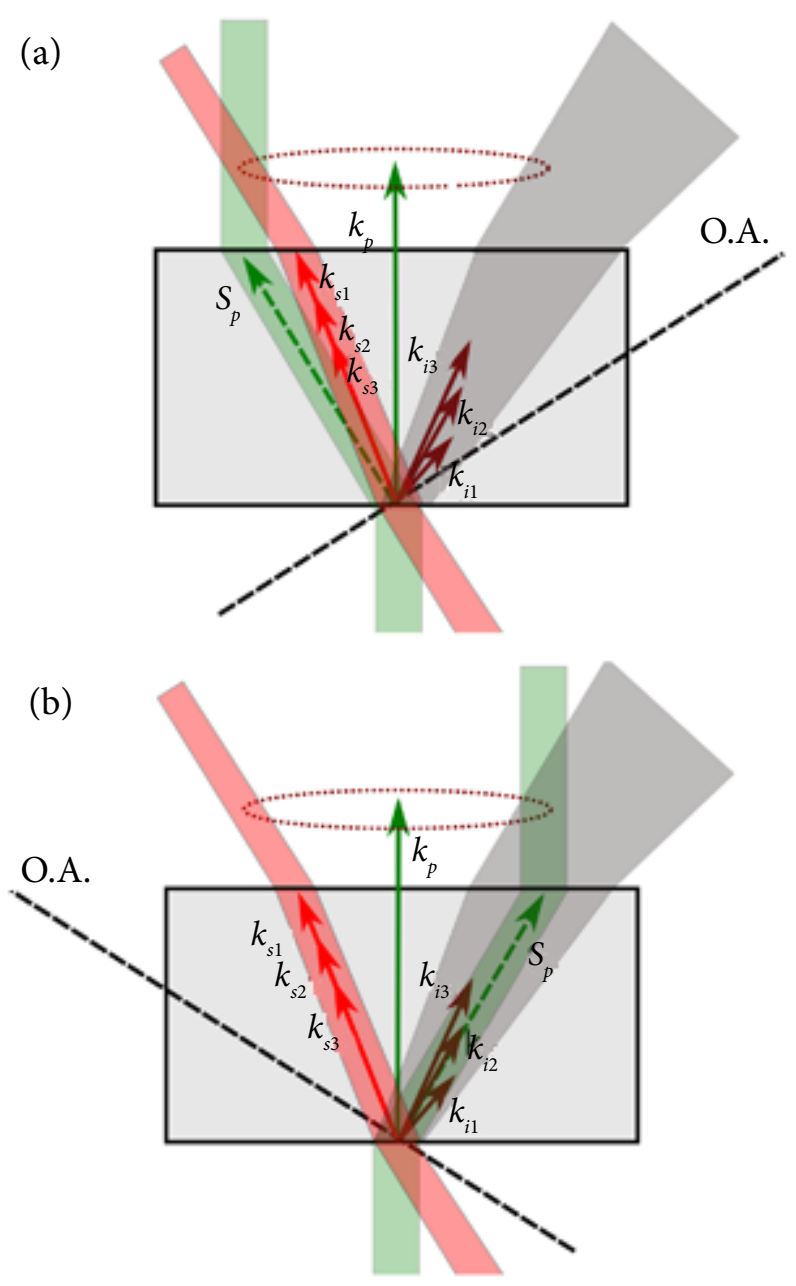

Fig. 1. Relative positions of the waves in Type I BBO crystal-based NOPA. (a) Poynting vector walk-off compensation geometry (PVWC), (b) tangential phasematching geometry (TPM). $k_{\mathrm{p}}, k_{\mathrm{s}}$, and $k_{\mathrm{i}}$ are wavevectors of pump, signal and idler waves, respectively. $S_{\mathrm{p}}$ is the pump beam Poynting vector. Signal and idler waves are indicated by sets of wavevectors representing different spectral components of broadband pulses. O. A. is the optical axis of the crystal. The dotted circles indicate the APF cone. The angles between the vectors are exaggerated for clarity.

geometry is called Poynting vector walk-off compensation geometry (PVWC) [15]. In the case of the other orientation of the optical axis (see Fig. 1(b)) - tangential phase-matching geometry (TPM) - the pump beam propagates very close to that of the angular dispersed idler beam. Both geometries are commonly used in noncollinear optical parametric amplifiers and allow for similar pump-to-signal conversion efficiencies. However, the spatial and spectral parameters of the amplified signal differ appreciably due to specific spatio-temporal pulse shaping dynamics and different parasitic frequency mixing processes in PVWC and TPM geometries. The PVWC geometry offers a much better near-field profile of the signal beam, however, the signal is accompanied by the parasitic second harmonic generation affecting the shape of the amplified spectrum [16]. On the other hand, numerical simulations have confirmed that undesirable spectral phase modulation of the signal pulse occurs in the TPM geometry [17]. Differences are more pronounced when narrow beams and short pulses are used in parametric amplifiers. As the contrast of an OPCPA system output is usually defined by the first amplification stage [13], the common practice is to design these first stages to operate with minimum beam sizes in order to enhance the ratio of seed and quantum noise photons in the amplification channel. Different spatio-temporal parametric amplification conditions in PVWC and TPM amplification configurations may also lead to different APF levels at the output of OPA. However, to the best of our knowledge, this issue has not yet been examined.

In this work we investigate the properties of APF produced in a high-gain BBO-based femtosecond noncollinear optical parametric amplifier (NOPA) pumped at $515 \mathrm{~nm}$ and examine the differences of APF levels in two different amplification geometries.

\section{Theoretical modelling}

\subsection{Governing equations}

The computer simulations of noncollinear parametric amplification were performed using nonlinear coupling equations that in the Fourier domain can be expressed as

$$
\begin{aligned}
& \frac{\partial^{2}}{\partial z^{2}} S_{1}+\left(k^{2}\left(\omega_{1}\right)-k_{x}^{2}-k_{y}^{2}\right) S_{1}=-\mu_{0} \omega_{1}^{2} \hat{P}_{1}, \\
& \frac{\partial^{2}}{\partial z^{2}} S_{2}+\left(k^{2}\left(\omega_{2}\right)-k_{x}^{2}-k_{y}^{2}\right) S_{2}=-\mu_{0} \omega_{2}^{2} \hat{P}_{2}, \\
& \frac{\partial^{2}}{\partial z^{2}} S_{3}+\left(k^{2}\left(\omega_{3}, k_{x}, k_{y}\right)-k_{x}^{2}-k_{y}^{2}\right) S_{3}=-\mu_{0} \omega_{3}^{2} \hat{P}_{3},
\end{aligned}
$$

where $j=1,2,3$ stands for signal, idler and pump waves, respectively. $S_{j}\left(\omega_{j}, k_{x}, k_{y}\right)$ are the Fourier transforms of electric fields $A_{j}(t, x, y) . k$ is a wave number and $k_{x}, k_{y}$ are the projections of the wave vector. We consider the Cartesian coordinates $(x, y, z)$. Time and cyclic frequency are denoted by $t$ and $\omega$, respectively. $\mu_{0}$ is the vacuum permeability and $\hat{P}_{j}$ is the Fourier transform of a nonlinear polarization.

We investigated the nonlinear Type I interaction in the BBO crystal. The signal and idler waves are ordinary and their wave numbers depend only on cyclic frequency $\omega$. The wave number of the pump wave 
depends also on the propagation direction (projections $k_{x}$ and $k_{v}$ ).

From Eq. (1) it follows that

$$
\begin{aligned}
& \frac{\partial}{\partial z} S_{1}=\mathrm{i} k_{z 1} S_{1}-\frac{\mu_{0} \omega_{1}^{2}}{2 \mathrm{i} k_{z 1}} \hat{P}_{1}, \\
& \frac{\partial}{\partial z} S_{2}=\mathrm{i} k_{z 2} S_{2}-\frac{\mu_{0} \omega_{2}^{2}}{2 \mathrm{i} k_{z 2}} \hat{P}_{2}, \\
& \frac{\partial}{\partial z} S_{3}=\mathrm{i} k_{z 3} S_{3}-\frac{\mu_{0} \omega_{3}^{2}}{2 \mathrm{i} k_{z 3}} \hat{P}_{3},
\end{aligned}
$$

where $k_{z 1,2}=\sqrt{k\left(\omega_{1,2}\right)^{2}-k_{x}^{2}-k_{y}^{2}}$ and

$$
k_{z 3}=\sqrt{k\left(\omega_{3}, k_{x}, k_{y}\right)^{2}-k_{x}^{2}-k_{y}^{2}} .
$$

Equation (2) was solved by a Fourier split-step method. The linear part was solved in the Fourier domain:

$$
S_{j}\left(z+h_{z}\right)=S_{j}(z) \exp \left(\mathrm{i} k_{z j} h_{z}-\mathrm{i} h_{z}\left(\omega_{j}-\omega_{j 0}\right) / u_{3}\right),
$$

where $h_{z}$ is the longitudinal step. The second term in the brackets was subtracted in order to reduce the time walk-off. We set $u_{3}=c / 1.65$, where $c$ is the speed of light. The nonlinear part was solved for the fields $A_{j}$ which were found by the use of the inverse Fourier transform of $S_{j}$. The simplifications were performed applying the paraxial approximation for the wave packets: the factors $\mu_{0} \omega_{j}^{2} /\left(2 \mathrm{i} k_{z j}\right)$ in the nonlinear terms were substituted by their values at $\omega_{j}=\omega_{j 0}, k_{x}=k_{y}=0$, where $\omega_{j 0}$ is the central frequency of the $j$ th wave. $k_{x}=$ $k_{y}=0, k_{z j}=k_{z j 0}$.

In order to describe quantum noise fluctuations we added the Langevin noise terms $\eta_{j}$. First, we note that $A_{j}=B_{j} \exp \left(\mathrm{i} k_{z j 0} z\right)$. The nonlinear and noise part of the equations was solved for amplitudes $B_{j}$ as follows:

$$
\begin{aligned}
& B_{1}\left(z+h_{z}\right)=B_{1}(z)+h_{z}\left[-\Gamma B_{1}(z)\right. \\
& \left.+\sigma_{1} B_{2}^{*}(z) B_{3}(z) \exp (\mathrm{i} \Delta k z)+\xi_{1} \sqrt{2 \Gamma / h_{z}}\right], \\
& B_{2}\left(z+h_{z}\right)=B_{2}(z)+h_{z}\left[-\Gamma B_{2}(z)\right. \\
& \left.+\sigma_{2} B_{1}^{*}(z) B_{3}(z) \exp (\mathrm{i} \Delta k z)+\xi_{2} \sqrt{2 \Gamma / h_{z}}\right], \\
& B_{3}\left(z+h_{z}\right)=B_{3}(z)+h_{z}\left[-\Gamma B_{3}(z)\right. \\
& \left.-\sigma_{3} B_{1}^{*}(z) B_{2}(z) \exp (-\mathrm{i} \Delta k z)+\xi_{3} \sqrt{2 \Gamma / h_{z}}\right] .
\end{aligned}
$$

Here $\Delta k=k_{z 30}-k_{z 20}-k_{z 10}$ is the phase mismatch. We note that the Langevin noise term $\eta_{j}$ is $\delta$-correlated:

$$
\begin{aligned}
& \left\langle\eta_{j}(t, x, y, z) \eta_{j}^{*}\left(t^{\prime}, x^{\prime}, y^{\prime}, z^{\prime}\right)\right\rangle \\
& =\eta_{0} \delta\left(x-x^{\prime}\right) \delta\left(y-y^{\prime}\right) \delta\left(t-t^{\prime}\right) \delta\left(z-z^{\prime}\right) .
\end{aligned}
$$

$\eta_{0}$ is the strength of noise. In Eq. (4) $\xi_{j}=\xi_{j}(x, y, t)$ is the normal random numbers with the variance $\eta_{0}$. $\Gamma$ describes the linear absorption. In the BBO crystal, $\Gamma=0.01 \mathrm{~cm}^{-1}$. Nonlinear coupling coefficients are described by

$$
\sigma_{j}=\frac{\omega_{z j 0}^{2} d^{\mathrm{eff}}}{c^{2} 2 k_{z j 0}},
$$

where $d^{\text {eff }}$ is the effective nonlinear susceptibility.

Equations (2) were simulated for Gaussian pulses with the following boundary conditions at $z=0$ :

$$
\begin{aligned}
& A_{1}=\alpha_{10} \exp \left(-4(1+\mathrm{i} \gamma) \frac{t^{2}}{\tau^{2}}-\frac{x^{2}+y^{2}}{\rho_{1}^{2}}\right) \exp \left( \pm \mathrm{i} k_{x 10} x\right) \\
& +\xi_{10} \\
& A_{2}=\xi_{20} \\
& A_{3}=\alpha_{30} \exp \left(-\frac{t^{2}}{\tau^{2}}-\frac{x^{2}+y^{2}}{\rho_{3}^{2}}\right)
\end{aligned}
$$

Here $\tau$ and $\rho$ are the pulse duration and beam radius, respectively. $k_{x 10}$ is the $x$ projection of the input signal beam wavevector. The sign ' + ' before it corresponds to PVWC amplification configuration, i. e. the pump walk-off towards the seed beam direction (see Fig. 1(a)). Sign '-' corresponds to TPM geometry (see Fig. 1(b)). $a_{10}$ and $a_{30}$ are the amplitudes of signal and pump pulsed beams, respectively. $\gamma$ is the chirp parameter. $\xi_{1,20}=\xi_{1,20}(x, y, t)$ is the input noise. Computer simulations were performed using the wavelength, beam size, pulse duration and pulse energy values corresponding to those used in the experiment. However, Gaussian pulse and beam shapes were assumed and no attempt was made to replicate the experimental conditions exactly. As we can see from Eq. (7), the input signal wave is a chirped pulse. The pump wavelength was set to $\lambda_{30}=515 \mathrm{~nm}$. We assume the noncollinearity angle for the signal beam to be $\alpha_{10}=2.56^{\circ}$ which was calculated by the use of Selmeier equations from [18]. The phase matching angle is $\theta_{3}=24.5^{\circ}$ and the central signal wavelength is $\lambda_{10}=795 \mathrm{~nm}$. The signal pulse chirp parameter is $\gamma=7.5 . \rho_{1}$ and $\rho_{3}$ are 250 and $110 \mu \mathrm{m}$ at FWHM, respectively. The pulse duration $\tau$ corresponds to $200 \mathrm{fs}$ at FWHM. The crystal length is $2.5 \mathrm{~mm}$ and the input seed energy is $1 \mathrm{~nJ}$. We also involved into consideration the nonlinear interaction length $L_{n j}=1 /\left(\alpha_{30} \sigma_{j}\right)$. For the $2 \mu \mathrm{J}$ pump energy (85 GW/ $\mathrm{cm}^{2}$ intensity) we obtain $L_{\mathrm{n} 3}=130 \mu \mathrm{m}$. $d^{\text {eff }}$ was taken from [19].

For evaluation of the input noise intensity we use the formula from Ref. [11] 


$$
I_{\text {noise }}=\frac{\hbar \omega^{3} n^{3}}{8 \pi^{3} c^{2}} \Delta \Omega \Delta \omega
$$

where $\hbar$ is the Plank constant and $n$ is the refractive index. $\Delta \omega$ is the detected spectral width and $\Delta \Omega$ is the solid angle of the emitted radiation. In the formula we have omitted an additional factor of 2 since we calculate signal and idler noises separately. For the wavelength window from $650 \mathrm{~nm}$ to $950 \mathrm{~nm}$ and the solid angle calculated from the divergence angle of the pump we obtain $I_{\text {noise }}=2180 \mathrm{~W} / \mathrm{m}^{2}$ that for the pulse duration of $200 \mathrm{fs}$ corresponds to only 20 photons. We have to magnify the obtained values by $N_{\theta}^{2}=1.7 \times 10^{3}$ since we consider the angular spectrum of the input signal noise with the divergence angle $2.5^{\circ}$ which is $N_{\theta}$ times larger than the divergence angle of the pump beam.

\subsection{Results of numerical simulations}

First, we have performed the modelling of parametric fluorescence amplification, i. e. the case when no seed is present at the OPA input. The angular spectrum of the APF signal beam is presented in Fig. 2. It was integrated over all frequency components. As we can see, the left side of the APF ring is brighter than the right one, though the pump walk-off for this case is on the opposite side with reference to the cone axis, i. e. corresponds to the situation depicted in Fig. 1. 1(b). This non-intuitive result can be explained by the fact that in this case the propagation direction of the idler beam nearly coincides with the pump beam. The propagation angle of the idler is larger than that of the signal, so it can better follow the pump beam during its walk-off.

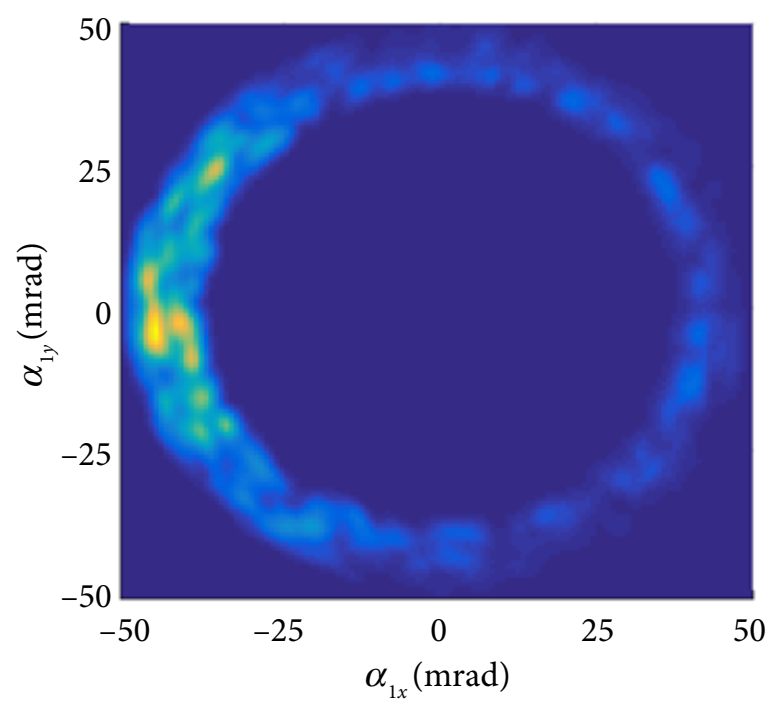

Fig. 2. Angular spectrum of APF. Pump intensity $170 \mathrm{GW} / \mathrm{cm}^{2}$.
In order to evaluate the amplified signal energy contrast with reference to APF we have repeated calculations of angular output radiation distribution in the presence of seed. The seed is directed into the crystal at the internal non-collinearity angle $\alpha_{10}=2.56^{\circ} \approx 45 \mathrm{mrad}$. PVWC and TPM configurations were examined simply by taking positive or negative $\alpha_{10}$ values, i. e. choosing $+k_{x 10}$ or $-k_{x 10}$ in Eq. (7). The light energy variation on the cone obtained by scanning it with a rectangular spatial aperture with a size of $18 \mathrm{mrad}$ is depicted in Fig. 3. The red curve (1) which shows the APF angular distribution when seed is absent (see Fig. 2) indicates that APF intensity on the right and left sides of the cone (see the points at 0 and 180 deg angles) differs by about one order of magnitude (more than 10 times).

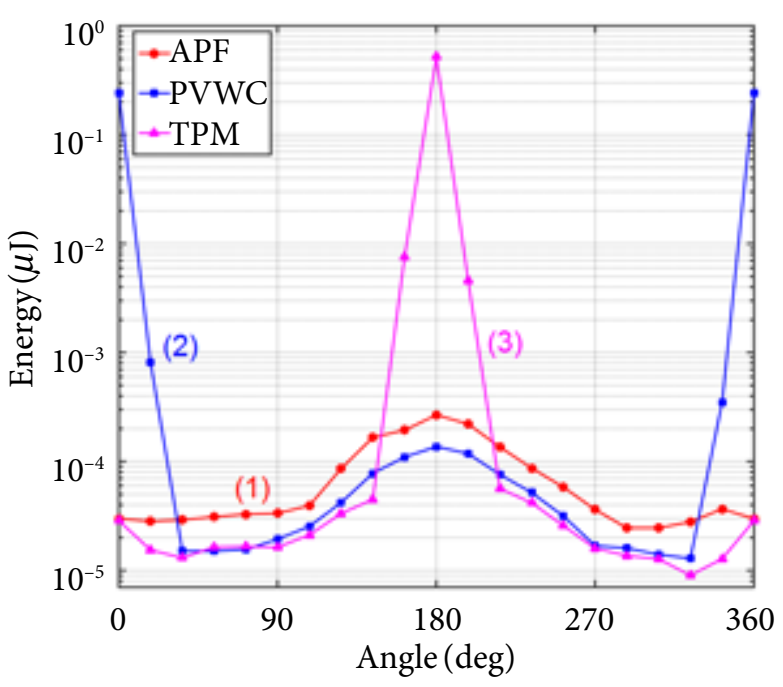

Fig. 3. Angular intensity distribution on the cone. Red (online) line 1 is APF energy when seed is blocked. Signal amplification in PVWC and TPM configurations is presented by blue (online) 2 and magenta (online) 3 lines, respectively. Pump intensity $170 \mathrm{GW} / \mathrm{cm}^{2}$.

When the seed is amplified in TPM geometry (the point on the magenta line at $180 \mathrm{deg}$ ), the amplified signal magnitude is higher than the APF level of an unseeded OPA (the point on the red line at $180 \mathrm{deg}$ ) by more than three orders of magnitude. The contrast ratio in the case of amplification in PVWC geometry is definitely higher, as the amplified pulse energy is smaller by $\sim 1.2$ times but the APF level is lower by an order of magnitude (see points at 0 deg on the blue and red line, respectively) as compared to the case of amplification in the TPM configuration. We point out that introducing the seed results in lowering of light intensity on the cone both for PVWC and TPM geometries. This 
APF gain suppression is caused by pump depletion due to energy transfer to the signal and leads to overall APF levels lower by several times when the OPA is seeded, as compared to APF levels in an unseeded OPA.

The data on signal and APF amplification in PVWC and TPM geometries at various pump intensities are presented in Fig. 4. One can see that the PVWC configuration provides a notably higher ratio of the amplified signal energy with respect to the energy of APF in the amplification channel.

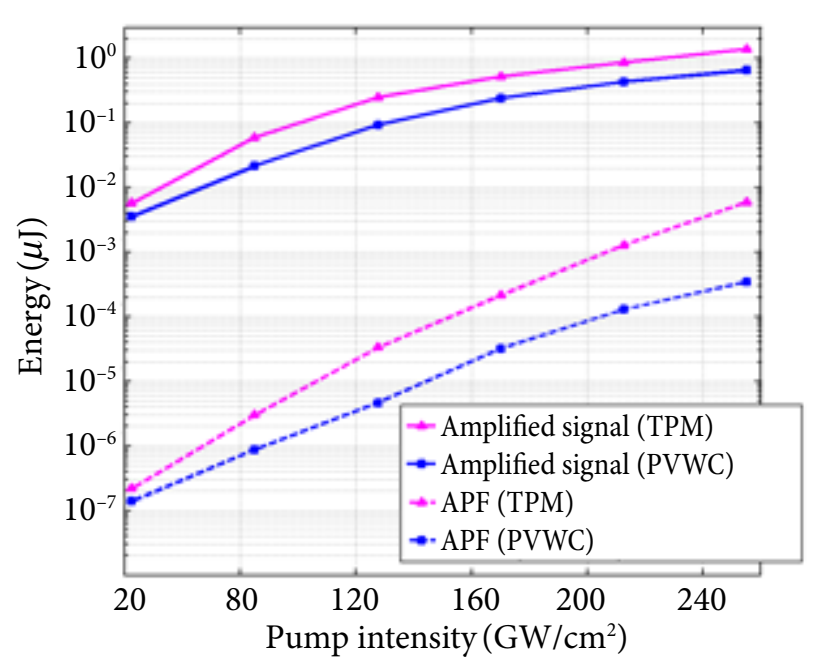

Fig. 4. Energies of the amplified signal (solid lines) and APF (dashed lines) for different pump intensities.

\section{Experiment}

In our setup a Yb:KGW diode-pumped solid state laser system (PHAROS, Light Conversion, Ltd.) generating $200 \mathrm{fs}$ pulses at $1030 \mathrm{~nm}$ at a repetition rate of $1 \mathrm{kHz}$ was used. A small part of the pulse energy $(\sim 1 \mu \mathrm{J})$ was split off and focused into a $4 \mathrm{~mm}$ sapphire plate to generate a white-light continuum (WLC) seed. A waveplate-polarizer attenuator was used to finely tune the pump energy for optimum WLC generation, which led to the WLC spectrum being highly stable on both shot-to-shot and day-to-day timescales. The larger part of the pulse energy was frequency doubled in a $0.7 \mathrm{~mm}$ BBO crystal, producing pump pulses for the NOPA. Parametric amplification was carried out in a $2.5 \mathrm{~mm}$ type-I BBO crystal into which the pump beam was focused to a spot size of $110 \mu \mathrm{m}$ at FWHM. The size of the seed beam was $250 \mu \mathrm{m}$ at FWHM. The phase matching angle of the crystal and the noncollinearity angle were fine-tuned for efficient signal amplification in the 670-950 nm spectral range. In our setup the continuum seed pulse with an energy content of $\sim 5 \mathrm{~nJ}$ in the 670-950 $\mathrm{nm}$ spectral band could be amplified up to $0.6 \mu \mathrm{J}$ at a pump intensity level of $170 \mathrm{GW} / \mathrm{cm}^{2}$ with a conversion efficiency of $11.5 \%$. Parasitic SHG at a wavelength of $435 \mathrm{~nm}$ was observed in the case of PVWC configuration. This results in a hole in the amplified spectrum at $\sim 870 \mathrm{~nm}$.

When the seed is blocked, the cone of APF is clearly observed at high pump intensity levels. The half-angle of the cone centered on the pump beam is set to $\sim 4.1^{\circ}(\approx 71 \mathrm{mrad})$ by tuning the phase matching angle of the crystal. Since the refraction index of the BBO crystal is $\sim 1.66$ at $800 \mathrm{~nm}$, this corresponds to an internal signal-pump noncollinearity angle of $\sim 2.5^{\circ}$ that is called the magic angle and provides the broadest spectral amplification band. An image of APF spatial distribution acquired with a beam profiling camera (WinCamD, DataRay Inc) is presented in Fig. 5(a). To avoid saturation of the detector, the pump beam had to be blocked with an appropriate dielectric mirror. Additionally, several calibrated neutral density filters were
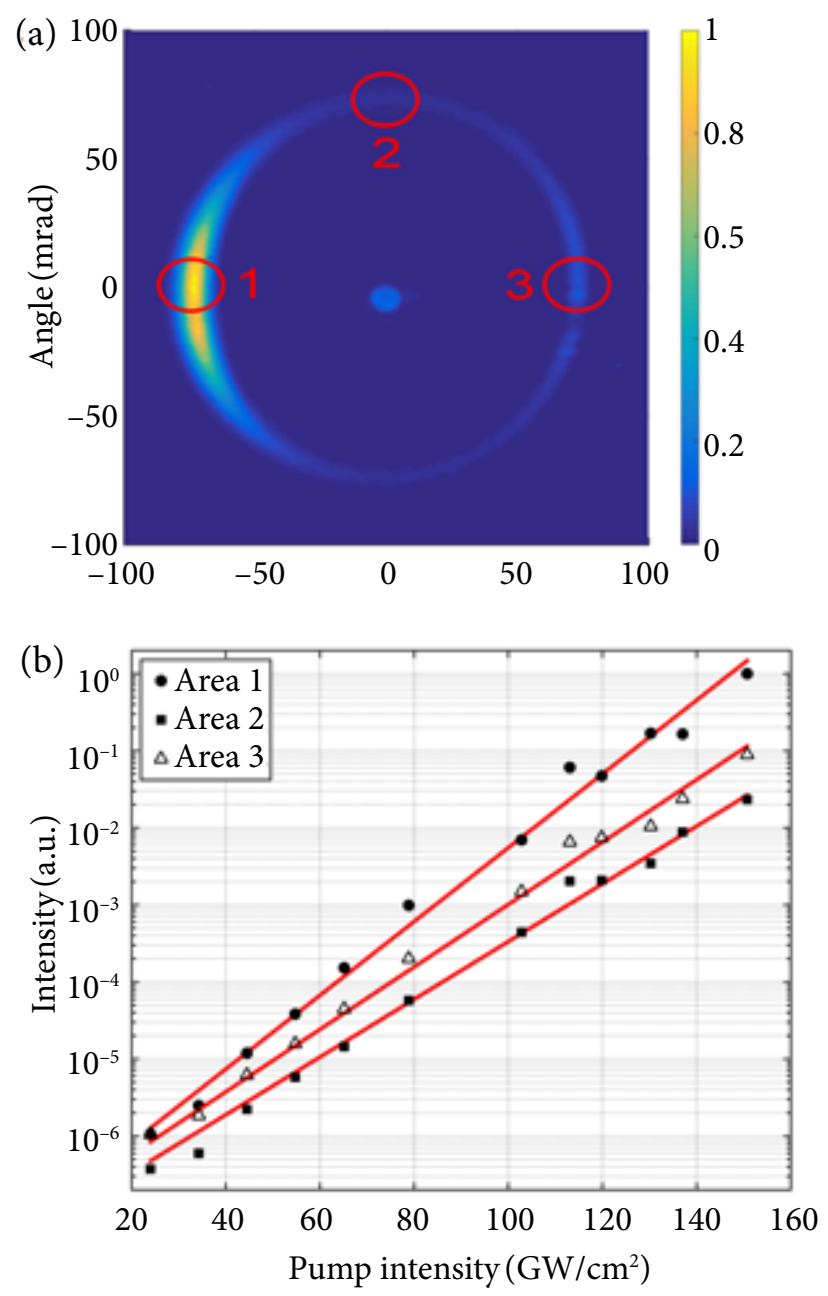

Fig. 5. An image of the SF cone at the output of NOPA (a) and the APF amplitude on different spatial zones of the cone (b) marked by (red online) circles in (a). 
used for measuring the superfluorescence at different levels of pumping. The difference between the APF amplitudes on the right and left sides of the cone arises due to varying the pump beam spatial overlap with an amplified signal or idler waves of parametric fluorescence. In this case the orientation of the optical axis corresponds to that which is shown in Fig. 1 (b).

We have measured the dependence of APF on pump intensity at different angles on the cone (1,2, 3 marked in Fig. 5(a)). The results presented in Fig. 5(b) show, as expected, the exponential APF intensity dependence on pump intensity for all three measured directions on the cone.

The amount of APF in the amplification channel measured by blocking the seed is the worst case assessment of the amplified pulse energy contrast. The presence of the seed at the input of OPA suppresses APF since even a small depletion of the pump pulse due to energy transfer to the injected signal reduces the amplification of parametric fluorescence [13]. To the best of our knowledge, the amount of APF suppression has so far only been measured in a single work [14] by creating a narrow spectral hole in the seed spectrum using an acousto-optic pulse shaper. In contrast, we have examined the APF suppression by measuring the APF intensity at the point on the APF cone that was away from the signal amplification channel, i. e. the 2 nd zone in Fig. 1(a). The light was collected by a $400 \mu \mathrm{m}$ diameter fiber and measured using a compact spectrometer (AvaSpec 3648, Avantes). Spectra acquired with the seed blocked and unblocked at different pump intensity levels were integrated in the wavelength region of our interest $(670-950 \mathrm{~nm})$. The obtained results are presented in Fig. 6 (a). APF suppression up to 6 times was measured when pump-to-signal energy conversion reached $11.5 \%$ at a pump intensity of $170 \mathrm{GW} /$ $\mathrm{cm}^{2}$ (see Fig. 6(b)). These results are close to the APF suppression ratio obtained by computer simulations (see Fig. 3).

The results of theoretical treatment presented in Section 2 show that the level of APF in PVWC and TPM non-collinear parametric amplification geometries differs considerably. In order to verify this finding experimentally we have measured the energies of both the amplified signal and APF (by blocking the seed) for the both amplification geometries. In our experimental conditions the APF at low pump intensities is very weak and cannot be measured directly by our standard power meter. In order to evaluate APF energies we blocked the seed and measured the spectra of APF propagating in the signal amplification channel. The energies of APF were calculated by taking spectra integrals over a range of $670-950 \mathrm{~nm}$ and scaling it by a factor, which was found by comparing energy values
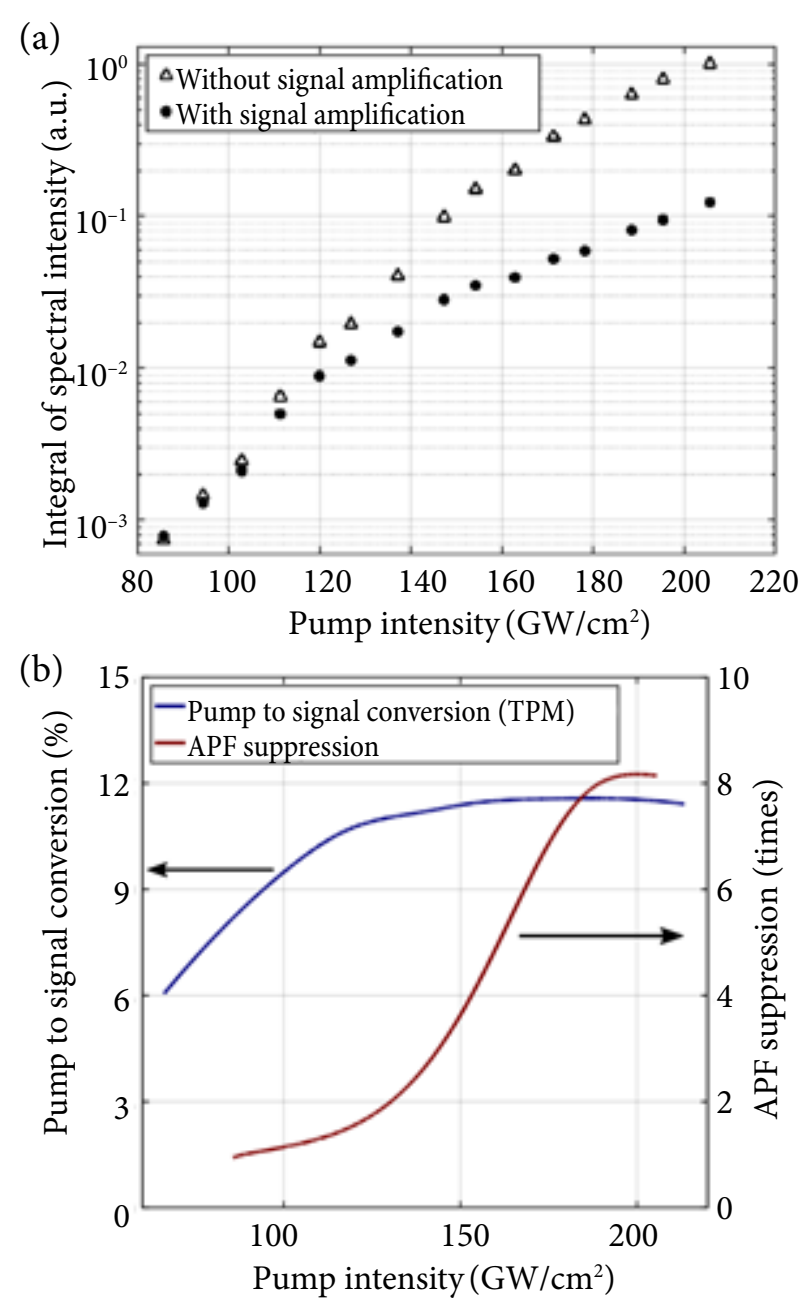

Fig. 6. APF amplitude with and without signal amplification (a); APF suppression and pump to signal conversion efficiency dependence on pump intensity (b).

measured with a spectrometer and a power meter at high pump intensity levels above $160 \mathrm{GW} / \mathrm{cm}^{2}$.

The obtained data are presented in Fig. 7 and show that one obtains a bit higher amplified signal energy in the TPM geometry. However, the APF energy level for different interaction geometries differs significantly and the APF energy content at the OPA output is less than $0.1 \%$ even in the strong amplification saturation regime in the PVWC configuration. When accounting for the APF suppression effect one can expect several times lower values of parametric fluorescence. The dashed lines in Fig. 7 show the expected levels of APF calculated by applying the suppression factor derived from the data presented in Fig. 6 (a). The amplified pulse contrast steadily drops with increasing pump intensity: seed amplification saturates, while the steadily rising contribution of APF from the temporal areas where the level of seed is low leads to a monotonous increase of an incoherent 


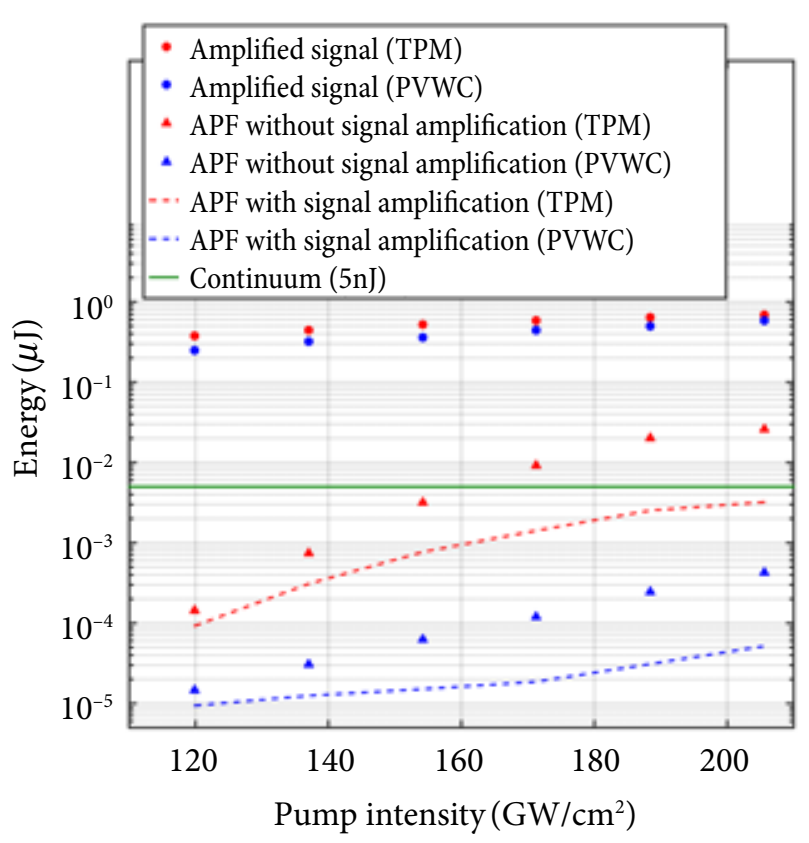

Fig. 7. Energies of the amplified signal and APF pulses for different amplification geometries and pump intensities.

background. Therefore, looking for the best trade-off between the high output energy and the low APF level, we consider the pump level of $140-170 \mathrm{GW} / \mathrm{cm}^{2}$ to be optimum. We also note that for pump levels above $180 \mathrm{GW} / \mathrm{cm}^{2}$ we observed undesirable modulations in the amplified pulse spectrum and the spatial profile caused by nonlinear self-action processes in the $\mathrm{BBO}$ crystal. The results of numerical modelling (see Section 2) show higher absolute values of the ratio of the amplified signal and APF as compared to the same ratios measured in the experiment. This can be explained by the fact that the computer simulations were performed for ideal wave packets with both spatial and temporal Gaussian profiles, while the characteristics of real pulses in the experiment were different to some extent. Nevertheless, the numerical and experimental results agree well qualitatively. It is seen that in the case of noncollinear parametric amplification of low intensity seed pulses the PVWC geometry has a considerable advantage over the TPM configuration in terms of a notably higher amplified pulse contrast.

\section{Conclusions}

We have investigated the properties of APF produced in a femtosecond NOPA that will serve as a front end in a TW-class OPCPA system [20] and examined the two possible geometric configurations of broadband noncollinear parametric amplification in the BBO crystal pumped by $515 \mathrm{~nm}$ pulses. The presented experimental and numerical results reveal that PVWC amplification geometry provides APF levels that are lower by an order of magnitude as compared to amplification in TPM geometry. Furthermore, we have used an original method to determine the level of APF suppression due to pump depletion by the signal and found it to be slightly smaller than 1 order of magnitude in typical OPA operating conditions. We believe that these findings are relevant for the optimization of OPCPA system designs for higher output pulse contrast.

\section{Acknowledgements}

This work was partially funded by the Research Council of Lithuania (Grant No. MIP-055/2014) and the EU 7th Framework Programme (Grant Agreement No. 284464).

\section{References}

[1] A. Dubietis, G. Jonušauskas, and A. Piskarskas, Powerful femtosecond pulse generation by chirped and stretched pulse parametric amplification in BBO crystal, Opt. Commun. 88, 437 (1992).

[2] J. Moses, C. Manzoni, S.-W. Huang, G. Cerullo, and F.X. Kärtner, Temporal optimization of ultrabroadband high-energy OPCPA, Opt. Express 17, 5540 (2009).

[3] J. Fülöp, Z. Major, A. Henig, S. Kruber, R. Weingartner, T. Clausnitzer, E. Kley, A. Tünnermann, V. Pervak, A. Apolonski, J. Osterhoff, R. Hörlein, F. Krausz, and S. Karsch, Short-pulse optical parametric chirped-pulse amplification for the generation of high-power few-cycle pulses, New J. Phys. 9, 438 (2007).

[4] T. Stanislauskas, R. Budriūnas, R. Antipenkov, A. Zaukevičius, J. Adamonis, A. Michailovas, L. Giniūnas, R. Danielius, A. Piskarskas, and A. Varanavičius, Table top TW-class OPCPA system driven by tandem femtosecond $\mathrm{Yb}$ : KGW and picosecond Nd: YAG lasers, Opt. Express 22, 1865 (2014).

[5] S. Harris, M. Oshman, and R. Byer, Observation of tunable optical parametric fluorescence, Phys. Rev. Lett. 18, 732 (1967).

[6] G.D. Boyd and D.A. Kleinman, Parametric interaction of focused Gaussian light beams, J. Appl. Phys. 39(8), 3597-3639 (1968).

[7] A. Laubereau, L. Greiter, and W. Kaiser, Intense tunable picosecond pulses in the infrared, Appl. Phys. Lett. 25, 87 (1974).

[8] R. Danielius, A. Piskarskas, A. Stabinis, G. Banfi, P. Di Trapani, and R. Righini, Traveling-wave parametric generation of widely tunable, highly coherent femtosecond light pulses, J. Opt. Soc. Am. B 10, 2222 (1993). 
[9] R. Glauber and F. Haake, The initiation of superfluorescence, Phys. Lett. A 68, 29 (1978).

[10]C. Manzoni, J. Moses, F.X. Kärtner, and G. Cerullo, Excess quantum noise in optical parametric chirpedpulse amplification, Opt. Express 19, 8357 (2011).

[11]C. Homann and E. Riedle, Direct measurement of the effective input noise power of an optical parametric amplifier, Laser Photon. Rev. 7, 580 (2013).

[12]J. Chwedeńczuk and W. Wasilewski, Intensity of parametric fluorescence pumped by ultrashort pulses, Phys. Rev. A 78, 063823 (2008).

[13]F. Tavella, A. Marcinkevičius, and F. Krausz, Investigation of the superfluorescence and signal amplification in an ultrabroadband multiterawatt optical parametric chirped pulse amplifier system, New J. Phys. 8, 219 (2006).

[14]X. Gu, G. Marcus, Y. Deng, T. Metzger, C. Teisset, N. Ishii, T. Fuji, A. Baltuska, R. Butkus, V. Pervak, H. Ishizuki, T. Taira, T. Kobayashi, R. Kienberger, and F. Krausz, Generation of carrier-envelopephase-stable 2 -cycle $740-\mu \mathrm{J}$ pulses at $2.1-\mu \mathrm{m}$ carrier wavelength, Opt. Express 17, 62 (2009).

[15]A.L. Oien, I.T. McKinnie, P. Jain, N.A. Russell, D.M. Warrington, and L.A. Gloster, Efficient, low- threshold collinear and noncollinear $\beta$-barium borate optical parametric oscillators, Opt. Lett. 22, 859 (1997).

[16] J. Bromage, J. Rothhardt, S. Hädrich, C. Dorrer, C. Jocher, S. Demmler, J. Limpert, A. Tünnermann, and J. Zuegel, Analysis and suppression of parasitic processes in noncollinear optical parametric amplifiers, Opt. Express 19, 16797 (2011).

[17] T. Lang, A. Harth, J. Matyschok, T. Binhammer, M. Schultze, and U. Morgner, Impact of temporal, spatial and cascaded effects on the pulse formation in ultrabroadband parametric amplifiers, Opt. Express 21, 949 (2013).

[18]V.G. Dmitriev, G.G. Gurzadyan, and D.N. Nikogosyan, Handbook of Nonlinear Optical Crystals, Vol. 64 (Springer, 2013).

[19]A.V. Smith, SNLO Nonlinear Optics Code (Sandia National Laboratories, Albuquerque, NM, 2004) p. 1423.

[20]R. Budriūnas, T. Stanislauskas, and A. Varanavičius, Passively CEP-stabilized frontend for few cycle terawatt OPCPA system, J. Optics 17, 094008 (2015).

\title{
NEKOLINEARAUS OPTINIO PARAMETRINIO STIPRINTUVO KAUPINAMO ANTRA FEMTOSEKUNDINIO Yb:KGW LAZERIO HARMONIKA PARAMETRINĖS FLUORESCENSIJOS TYRIMAS
}

\author{
T. Stanislauskas, I. Balčiūnas, V. Tamulienè, R. Budriūnas, A. Varanavičius \\ Vilniaus universiteto Fizikos fakultetas, Kvantines elektronikos katedra, Vilnius, Lietuva
}

\begin{abstract}
Santrauka
Pristatomas tyrimas, skirtas surasti optimalias nekolinearaus optinio parametrinio stiprinimo sąlygas BBO kristale kaupinant femtosekundine $515 \mathrm{~nm}$ bangos ilgio spinduliuote, pagrindinị dèmesị skiriant toms sąlygoms, kurios leistų pasiekti geresni stiprinimo signalinio impulso kontrastą. Pasitelkus teorinị modeliavimą ir eksperimento rezultatus nustatyta, kad nepageidaujamos
\end{abstract}

sustiprintos parametrinès fluorescencijos (SPF) lygis gerokai skiriasi atsižvelgiant ị pasirinktą vieną iš dviejų galimų stiprinimo geometrijų. Be to, nauju metodu palyginus SPF intensyvumą vykstant užkrato stiprinimui ir be jo, nustatyta, kad kaupinimo nuskurdinimo atveju SPF lygis sumažeja 6 ir daugiau kartų, kai keitimo iš kaupinimo ị signalą efektyvumas pasiekia $11,5 \%$. 